\title{
Prognostic factors in patients with recurrent hepatocellular carcinoma treated with salvage liver transplantation: a single- center study
}

\author{
Pusen Wang ${ }^{1, *}$, Hao $\mathrm{Li}^{1, *}$, Baojie Shi ${ }^{1}$, Weitao Que ${ }^{1}$, Chunguang Wang ${ }^{1}$, Junwei \\ Fan' ${ }^{1}$, Zhihai Peng ${ }^{1}$, Lin Zhong ${ }^{1}$ \\ ${ }^{1}$ Department of General Surgery, Shanghai General Hospital, Shanghai Jiao Tong University School of Medicine, 200080, \\ Shanghai, China \\ *These authors have contributed equally to this work \\ Correspondence to: Zhihai Peng, e-mail: pengzh_doctor@hotmail.com \\ Lin Zhong, e-mail: zhongl_3284@hotmail.com
}

Keywords: hepatocellular carcinoma, liver resection, prognosis, recurrence, salvage liver transplantation

Received: February 02, 2016

Accepted: April 16, 2016

Published: April 27, 2016

\section{ABSTRACT}

\begin{abstract}
Although salvage liver transplantation (LT) has been widely adopted as a treatment for recurrent hepatocellular carcinoma(HCC), candidate selection criteria have not been established. This single-center study aimed to identify risk factors associated with HCC recurrence and survival following salvage LT. The study included 74 patients treated with salvage LT between October 2001 and February 2013. The median follow-up was $\mathbf{3 7 . 2}$ months after LT. There were 29 cases of HCC recurrence and 31 deaths following LT. Microvascular invasion at the time of liver resection, a time interval to post-LR HCC recurrence of $\leq 12$ months, an alpha-fetoprotein level at LT greater than $200 \mathrm{ng} / \mathrm{mL}$, and having undergone LT outside of the UCSF criteria were independent risk factors for HCC recurrence after salvage LT. Patients with no more than one risk factor had a 5-year recurrence-free survival rate of $71.2 \%$ compared to $15.9 \%$ in patients with two or more risk factors. These findings suggest that to avoid post-LT HCC recurrence and a dismal prognosis, patients with no more than one risk factor for recurrence should be given priority for salvage LT. These criteria may improve the outcomes of patients treated with salvage LT and facilitate the effective use of limited organ supplies.
\end{abstract}

\section{INTRODUCTION}

Hepatocellular carcinoma (HCC) is one of the most common neoplasms and the third leading cause of cancerrelated death worldwide [1]. There were an estimated 782,500 new liver cancer cases and 745,500 liver cancerrelated deaths in 2012, and HCC accounted for $70-90 \%$ of these cases. China accounted for approximately $50 \%$ of the total number of cases and deaths [2]. For patients with early-stage HCC, liver transplantation (LT) achieves the best outcomes ( 5 -year survival rate $>80 \%$ ) because it results in the complete removal of the tumor as well as treats the underlying chronic liver disease [3-5]. However, the shortage of donor organs is a major obstacle, particularly in China, due to the large population base, the donor risks associated with living-donor LT, the high cost of LT, and the associated legal limitations. This ultimately reduces the efficacy of this curative treatment [6].

Salvage LT is a strategy that offers liver resection (LR) followed by LT in patients with tumor recurrence or deteriorating liver function [7]. Previous studies have demonstrated improved survival outcomes in LR, possibly as a result of advanced surgical techniques, patient selection criteria, and postoperative management [8]. Given the shortage of donor organs and the typical progression of HCC (which can cause patients to drop out of the transplant waiting list), LR followed by salvage LT has become widely accepted. Furthermore, salvage LT, defined as LR performed to bridge patients to transplantation and avoid dropout, has been shown to be highly effective, with short- and long-term postoperative survival outcomes comparable to upfront LT [9-15]. 
HCC recurrence, which is fatal in almost all cases, is still the most important negative predictor of post-LT survival [15]. Over the past few years, substantial efforts have been made to select patients who have a low risk of recurrence, with the aim of curing as many HCC patients as possible [16]. As a result, the Milan, University of California San Francisco (UCSF), and Up-to-Seven (Up-to-7) selection criteria have been proposed. Patients with tumors that meet these criteria have been shown to have a lower rate of postLT HCC recurrence [3, 17, 18]. Nonetheless, the salvage policy differs from that of primary LT since the former can provide pathological data at LR. This could help predict recurrence, both post-LR [19] and post-salvage LT [20]. However, recurrent cases of HCC exhibited remarkably different clinical behavior despite meeting these criteria [21]. Therefore, the use of only the Milan, UCSF, or Upto-7 criteria, all of which consider tumor size and number as selection criteria, is not sufficient for salvage LT.

In this retrospective study, we aimed to identify risk factors for HCC recurrence, recurrence-free survival (RFS), and overall survival (OS) after salvage LT in order to improve salvage LT strategies and outcomes, and to help establish proper candidate selection criteria.

\section{RESULTS}

\section{Patient characteristics}

The characteristics of the 74 patients at LR and LT are summarized in Tables 1 and 2, respectively. The participants were predominantly male $(n=68 ; 91.9 \%)$. The underlying liver diseases in the study population were hepatitis B virus (HBV) $(n=68)$, hepatitis $C$ virus $(\mathrm{HCV})$ $(n=2)$, and autoimmune hepatitis $(n=4)$. At the time of the initial LR, 19 patients had an alpha-fetoprotein (AFP) level $>200 \mathrm{ng} / \mathrm{mL}, 57$ patients were within the Milan criteria, 48 patients had an Edmondson grade of 1-2, and microvascular invasion (MVI) was detected in 23 patients. Recurrence occurred $>12$ months post-LR in 46 patients and $\leq 12$ months post-LR in 28 patients.

The mean participant age at the time of salvage LT was 48.9 years, while the mean pre-LT Model for End-Stage Liver Disease (MELD) score was 10.8. Recurrence occurred within the UCSF criteria in 57 cases and outside the criteria in 17 cases. At the time of salvage LT, 62 patients had Child-Pugh grade A, 12 patients had an alpha-fetoprotein (AFP) level > $200 \mathrm{ng} / \mathrm{mL}$, and MVI was detected in 29 patients. The median follow-up after LT was 37.2 (range, 2.3-81) months. A total of 29 cases of HCC recurrence and 31 deaths during LT follow-up were recorded.

\section{Risk factors for $\mathrm{HCC}$ recurrence after salvage $\mathbf{L T}$}

Univariate and multivariate analyses were performed to identify risk factors for HCC recurrence after salvage LT (Table 3). On univariate analysis, the strongest pre-LT predictors of recurrence were HCC status outside UCSF criteria at LT with an odds ratio (OR) of $8.33(95 \%$ confidence interval $[\mathrm{CI}]: 2.36-29.38, P<0.001)$, and a time interval to post-LR HCC recurrence of $\leq 12$ months $(\mathrm{OR}=5.73 ; 95 \% \mathrm{CI}: 2.05-16.01, P=0.001)$. The other risk factors significantly associated with HCC recurrence were: advanced Edmondson grade at LR $(P=0.016)$, MVI at LR $(P=0.010)$, AFP level $>200 \mathrm{ng} / \mathrm{mL}$ at LT $(P=0.009)$, and advanced tumor node metastasis (TNM) staging at $\operatorname{LT}(P=0.029)$.

Variables with $P$ values $<0.10$ were subjected to multivariate logistic regression analysis, which revealed that MVI at LR (OR $=3.71 ; 95 \% \mathrm{CI}: 1.04-13.29, P=$ $0.044)$, a time interval to post-LR HCC recurrence of $\leq$ 12 months $(\mathrm{OR}=4.04 ; 95 \% \mathrm{CI}: 1.23-13.32, P=0.022)$, AFP level $>200 \mathrm{ng} / \mathrm{mL}$ at $\mathrm{LT}(\mathrm{OR}=6.53 ; 95 \% \mathrm{CI}: 1.17-$ $36.51, P=0.033$ ), and HCC status outside the UCSF criteria at LT $(\mathrm{OR}=6.12 ; 95 \% \mathrm{CI}: 1.39-26.92, P=$ 0.016) were independent risk factors for HCC recurrence following salvage LT.

\section{Prognostic factors affecting RFS and OS after salvage LT}

Univariate and multivariate analyses of patients who underwent salvage LT were performed to identify prognostic factors that affected RFS and OS. Univariate analyses using the Kaplan-Meier method and log-rank tests revealed that Edmondson grade 3-4 at LR ( $P=$ $0.011)$, the presence of MVI at LR $(P=0.003)$, a time interval to post-LR HCC recurrence of $\leq 12$ months $(P<$ $0.001)$, TNM stage 3-4 at LT $(P=0.018)$, AFP level $>$ $200 \mathrm{ng} / \mathrm{mL}$ at LT $(P=0.001)$, and HCC status outside the UCSF criteria at LT $(P<0.001)$ were identified as risk factors that were significantly associated with RFS after salvage LT. Edmondson grade $3-4$ at LR $(P=0.004)$, the presence of MVI at LR $(P=0.001)$, time interval to postLR HCC recurrence of $\leq 12$ months $(P=0.001)$, AFP level $>200 \mathrm{ng} / \mathrm{mL}$ at LT $(P=0.002)$, HCC status outside the UCSF criteria at LT $(P=0.001)$, and MELD score at $\mathrm{LT} \geq 15(P=0.005)$ were associated with OS. The $1-$, 3 -, and 5-year survival rates as well as $P$ values for the variables are shown in Table 4.

On multivariate Cox proportional hazard regression analysis, the presence of MVI at LR (hazard ratio [HR] = $3.10 ; 95 \% \mathrm{CI}: 1.44-6.68, P=0.004)$, time interval to postLR HCC recurrence of $\leq 12$ months $(\mathrm{HR}=3.25 ; 95 \% \mathrm{CI}$ : 1.41-7.49, $P=0.006)$, and HCC status outside the UCSF criteria at LT $(\mathrm{HR}=2.68 ; 95 \% \mathrm{CI}: 1.18-6.06, P=0.018)$ were identified as independent risk factors that affected RFS post-salvage LT. The presence of MVI at LR (HR $=4.47 ; 95 \% \mathrm{CI}: 1.97-10.14, P<0.001)$, time interval to post-LR HCC recurrence of $\leq 12$ months $(\mathrm{HR}=3.76 ; 95 \%$ CI: $1.78-7.94, P=0.001)$ and MELD score at LT $\geq 15$ affected $\mathrm{OS}(\mathrm{HR}=4.71,95 \% \mathrm{CI}: 2.08-10.71, P<0.001)$ 


\section{parameters}

$\operatorname{Sex}(n)$

Male

Female

Blood type (n)

A

B

AB

$\mathrm{O}$

Preoperative AFP level (n)

$>200 \mathrm{ng} / \mathrm{mL}$

$\leq 200 \mathrm{ng} / \mathrm{mL}$

Underlying liver disease (n)

Hepatitis B

Hepatitis C

Autoimmune hepatitis

HCC status

Within Milan criteria

Outside Milan criteria

Edmondson grade (n)

$1-2$

MVI (n)

Yes

No

Time interval to post-LR HCC recurrence (n)

$>12$ months

LR: liver resction; AFP: alphafetoprotein; HCC: hepatocellular carcinoma; MVI: microinvascular invasion.

(Table 5). Kaplan-Meier survival curves of RFS and OS according to each risk factor identified on multivariate analysis after salvage LT are shown in Figures 1 and 2, respectively.

\section{Prognosis after salvage LT according to number of risk factors}

On multivariate logistic regression analysis of postsalvage LT HCC recurrence, four independent factors were identified. Patients were divided into four groups according to number of risk factors. The risk of HCC recurrence progressively increased as the number of risk factors increased from 0,1 , or 2 to 3 or 4 as follows: $11.54 \%(\mathrm{n}=3 / 26), 33.33 \%(\mathrm{n}=9 / 27), 71.43 \%(\mathrm{n}=$ $10 / 14)$, and $100 \%(\mathrm{n}=7 / 7)$, respectively. Kaplan-Meier survival estimates for the four groups showed that when patients with no risk factors were used as a reference, the other three groups had significantly worse RFS $(P=0.026$, $P<0.001$, and $P<0.001$, respectively). The 1-, 3-, and 5 -year survival rates and mean RFS are also shown(Table 6 , Figure 3). We reported mean survival (Table 6) because there were no more than 50\% known deaths during followup in patients with $0-1$ risk factors, and therefore median 
Table 2: Clinicopathological characteristics of the patients at salvage LT

\begin{tabular}{|c|c|}
\hline Parameters & \\
\hline Age & $48.9 \pm 9.2$ \\
\hline Preoperative MELD score & $10.8 \pm 5.9$ \\
\hline \multicolumn{2}{|l|}{ Child-Pugh grading (n) } \\
\hline $\mathrm{A}$ & 62 \\
\hline B & 12 \\
\hline \multicolumn{2}{|l|}{ Preoperative AFP level (n) } \\
\hline$>200 \mathrm{ng} / \mathrm{mL}$ & 12 \\
\hline$\leq 200 \mathrm{ng} / \mathrm{mL}$ & 62 \\
\hline \multicolumn{2}{|l|}{ HCC status (n) } \\
\hline Within UCSF criteria & 57 \\
\hline Outside UCSF criteria & 17 \\
\hline \multicolumn{2}{|l|}{ TNM staging } \\
\hline $1-2$ & 56 \\
\hline $3-4$ & 18 \\
\hline \multicolumn{2}{|l|}{ MVI (n) } \\
\hline Yes & 29 \\
\hline No & 45 \\
\hline \multicolumn{2}{|c|}{ HCC recurrence after Salvage LT (n) } \\
\hline Yes & 29 \\
\hline No & 45 \\
\hline Follow-up after LT (months) & $37.2(2.3-81)$ \\
\hline Number of deaths (n) & 31 \\
\hline
\end{tabular}

LT: liver transplantation; MELD: model for end-stage liver disease; AFP: alphafetoprotein; HCC: hepatocellular carcinoma; UCSF: University of California San Francisco; TNM: tumor node metastasis; MVI: microinvascular invasion.

survival for these two cohorts was not reached in KaplanMeier analysis. Furthermore, two distinct subgroups of patients were defined: those with low risk (0-1 risk factors) and high risk (2-4 risk factors). The Kaplan-Meier survival curves showed a clear distinction between the two $\operatorname{groups}(P<0.001)$ (Figure 4).

\section{DISCUSSION}

We demonstrated that $\mathrm{HCC}$ recurrence post-salvage LT was not only dependent on UCSF criteria status, which considers tumor size and number, but was also affected by the biological characteristics of the tumor such as the presence of MVI at initial LR, the time interval to post-LR HCC recurrence, and the pre-LT AFP level. When any of these four independent risk factors were considered, the risk of recurrence increased significantly while RFS decreased compared to patients with no risk factors. Patients at low risk of recurrence had remarkably higher RFS than those at high risk $(71.2 \%$ vs. $15.9 \%$, respectively). Furthermore, MVI at LR, the time interval to post-LR HCC recurrence, and the MELD score at LT were independent risk factors in addition to the UCSF criteria that affected post-salvage LT survival. Based on these data, we conclude that patients with $0-1$ risk factors are better candidates for salvage LT than patients with $\geq$ 2 risk factors.

Given the donor liver shortage, salvage LT was proposed as an effective way to reduce dropout of patients waiting for a matched donor and delay tumor progression [7]. However, the candidate selection criteria for salvage LT are not well established. As a result of advances in surgical techniques and perioperative management over the past few decades, salvage LT is considered safe for patients deemed to be within the Milan [10], UCSF [14], and Up-to-7 [11] criteria. Additionally, the use of 
Table 3: Significant risk factors for HCC recurrence after salvage LT (univariate and multivariate analysis)

\begin{tabular}{lcccccc}
\hline & \multicolumn{3}{c}{ Univariate Analysis } & \multicolumn{2}{c}{ Multivariate Analysis } \\
\cline { 2 - 7 } Variables & OR & $\mathbf{9 5 \%}$ CI & $\boldsymbol{p}$ Value & OR & $\mathbf{9 5 \%}$ CI & $\boldsymbol{p}$ Value \\
\hline At LR & 1.62 & $0.30-8.61$ & 0.673 & & & \\
\hline Male sex & 1.00 & $0.13-4.44$ & 1.000 & & & \\
Hepatitis B virus & 0.60 & $0.49-0.72$ & 0.517 & & & \\
Hepatitis C virus & 1.59 & $0.21-11.98$ & 0.642 & & & \\
Autoimmune hepatitis & 1.18 & $0.41-3.40$ & 0.763 & & & \\
Pre-LR AFP level $>200 \mathrm{ng} / \mathrm{mL}$ & 2.08 & $0.69-6.25$ & 0.186 & & & \\
Outside Milan criteria & 3.31 & $1.22-8.97$ & 0.016 & 3.21 & $0.77-13.51$ & 0.044 \\
Advanced Edmondson grade & 3.73 & $1.33-10.47$ & 0.010 & 3.71 & $1.04-13.29$ & 0.022 \\
MVI presence & 5.73 & $2.05-16.01$ & 0.001 & 4.04 & $1.23-13.32$ & \\
Post-LR HCC recurrence $\leq 12$ months & & & & & & \\
\hline At LT & 1.11 & $0.43-2.85$ & 0.83 & & & \\
\hline Age $>50$ y & 1.11 & $0.37-3.36$ & 0.85 & & & \\
Pre-LT MELD score $\geq 15$ & 3.85 & $0.78-20.00$ & 0.098 & 3.83 & $0.48-30.30$ & 0.203 \\
Child-Pugh score $\geq 7$ & 6.30 & $1.54-25.83$ & 0.009 & 6.53 & $1.17-36.51$ & 0.033 \\
Pre-LT AFP level $>200 \mathrm{ng} / \mathrm{mL}$ & 8.33 & $2.36-29.38$ & $<0.001$ & 6.12 & $1.39-26.92$ & 0.016 \\
Outside UCSF criteria & 3.32 & $1.10-9.98$ & 0.029 & 1.66 & $0.36-7.65$ & 0.513 \\
Advanced TNM staging & & & & & & \\
\hline
\end{tabular}

HCC: hepatocellular carcinoma; LT: liver transplantation; LR: liver resction; MVI: microinvascular invasion; MELD: model for end-stage liver disease; AFP: alphafetoprotein; UCSF: University of California San Francisco; TNM: tumor node metastasis; OR: odds ratio; CI: confidence interval.

laparoscopic LR has rapidly increased, which has reduced the rate of adhesions that occur near the intestine and omentum, and made subsequent LT much safer when performed by specialist physicians [15]. Furthermore, salvage LT was different when pathologic data were provided at initial LR. Considering all of these factors, more studies are needed to establish the appropriate selection criteria for salvage LT that could supplement the Milan, UCSF, and Up-to-7criteria.

The presence of MVI, an aggressive HCC phenotype, is associated with poor prognosis [22]. In our cohort, 23 patients had MVI at LR compared to 29 at LT. These results were consistent with those of a previous study that reported increased MVI ratios at the time of the second surgery [23]. The presence of MVI at LR was identified as an independent risk factor for recurrence and decreased survival after salvage LT. We observed differences in the MVI results from the two pathological examinations. There were five cases $(6.8 \%)$ in which MVI status shifted from positive to negative and $11(14.9 \%)$ in which MVI shifted from negative to positive. However, $18(18 / 23)$ patients had MVI at both initial LR and salvage LT, suggestive of a potential correlation in MVI status between at LR and at LT. The presence of MVI in the explanted liver, a strong risk factor for recurrence, was not included in the analysis because it was not clinically relevant to predict post-LT survival at a time point before salvage LT with a variable that only becomes evident postLT [24].

Lee et al. suggested that patients with microscopic portal vein invasion at initial LR may not be candidates for salvage LT [20]. Additionally, they found that patients with progressive tumors were more likely to have MVI of the portal vein and had a higher incidence of early recurrence ( $\leq 12$ months). Another identified risk factor, time interval to post-LR HCC recurrence of $\leq 12$ months, is reportedly an indication of primary tumor metastasis, while late recurrence is indicative of multi-centric occurrence [25]. Therefore, even in cases of transplantable recurrent HCC(according to the UCSF criteria), there might be a risk of including tumors that originated from metastasis of the primary tumor [20]. Similarly, Hu et al. reported that a time interval to tumor recurrence of $<12$ months was an independent predictor for OS in patients who underwent salvage LT [26]. Lee et al. 
Table 4: Variables significantly related to post salvage LT survival (univariate analysis)

\begin{tabular}{|c|c|c|c|c|c|c|c|c|c|c|}
\hline \multirow[b]{3}{*}{ Variables } & \multirow{2}{*}{\multicolumn{3}{|c|}{$\begin{array}{c}\text { Recurrence-free } \\
\text { Survival (\%) } \\
\end{array}$}} & \multirow{3}{*}{ HR (95\% CI) } & \multirow{3}{*}{$p$ Value } & \multirow{2}{*}{\multicolumn{3}{|c|}{ Overall Survival (\%) }} & \multirow{3}{*}{ HR $(95 \%$ CI) } & \multirow{3}{*}{$p$ Valuc } \\
\hline & & & & & & & & & & \\
\hline & $1 y$ & $3 \mathbf{y}$ & $5 y$ & & & $1 \mathrm{y}$ & $3 y$ & $5 y$ & & \\
\hline \multicolumn{11}{|l|}{$\begin{array}{l}\text { Edmondson grade } \\
\text { at LR }\end{array}$} \\
\hline $1-2$ & 82.9 & 75.3 & 65.9 & $2.51[1.20-5.24]$ & 0.011 & 85.4 & 78.3 & 66.0 & $2.78[1.36-5.68]$ & 0.004 \\
\hline $3-4$ & 79.7 & 37.3 & 32.0 & & & 84.3 & 58.7 & 13.3 & & \\
\hline \multicolumn{11}{|l|}{ MVI at LR } \\
\hline Yes & 60.9 & 42.5 & 37.2 & $2.84[1.37-5.91]$ & 0.003 & 73.9 & 51.0 & 26.8 & $3.35[1.61-6.99]$ & 0.001 \\
\hline No & 91.5 & 71.4 & 62.4 & & & 89.9 & 80.7 & 58.4 & & \\
\hline \multicolumn{11}{|l|}{$\begin{array}{l}\text { Post-LR HCC } \\
\text { recurrence }\end{array}$} \\
\hline$>12$ months & 88.7 & 74.2 & 70.9 & $3.85[1.81-8.18]$ & $<0.001$ & 91.1 & 83.3 & 66.5 & $3.05[1.48-6.29]$ & 0.001 \\
\hline$\leq 12$ months & 70.1 & 41.3 & 26.7 & & & 75.0 & 52.5 & 24.4 & & \\
\hline \multicolumn{11}{|l|}{$\begin{array}{l}\text { TNM staging at } \\
\text { LT }\end{array}$} \\
\hline $1-2$ & 85.0 & 71.5 & 60.7 & $2.43[1.14-5.17]$ & 0.018 & 83.6 & 75.7 & 57.6 & $1.84[0.88-3.86]$ & 0.098 \\
\hline $3-4$ & 72.2 & 34.2 & 34.2 & & & 88.9 & 58.2 & 23.3 & & \\
\hline \multicolumn{11}{|l|}{ AFP level at LT } \\
\hline$>200 \mathrm{ng} / \mathrm{mL}$ & 66.7 & 40.0 & 13.3 & $3.38[1.52-7.49]$ & 0.001 & 75.0 & 31.3 & 20.8 & $3.29[1.48-7.32]$ & 0.002 \\
\hline$\leq 200 \mathrm{ng} / \mathrm{mL}$ & 84.8 & 66.6 & 61.5 & & & 87.0 & 79.7 & 53.5 & & \\
\hline \multicolumn{11}{|l|}{ HCC status at LT } \\
\hline $\begin{array}{l}\text { Within UCSF } \\
\text { criteria }\end{array}$ & 87.4 & 74.4 & 66.7 & $4.47[2.23-9.42]$ & $<0.001$ & 87.6 & 79.3 & 61.5 & $3.25[1.59-6.66]$ & 0.001 \\
\hline $\begin{array}{l}\text { Outside UCSF } \\
\text { criteria }\end{array}$ & 62.6 & 20.9 & 10.4 & & & 76.0 & 44.3 & 10.1 & & \\
\hline \multicolumn{11}{|l|}{$\begin{array}{l}\text { MELD score at } \\
\text { LT }\end{array}$} \\
\hline$\geq 15$ & & & & & & 70.6 & 39.2 & 29.4 & $2.79[1.32-5.89]$ & 0.005 \\
\hline$<15$ & & & & & & 89.4 & 81.3 & 52.3 & & \\
\hline
\end{tabular}

LT: liver transplantation; LR: liver resction; MVI: microinvascular invasion; HCC: hepatocellular carcinoma; TNM: tumor node metastasis; AFP: alphafetoprotein; UCSF: University of California San Francisco; MELD: model for end-stage liver disease; HR: hazard ratio; CI: confidence interval.

Table 5: Variables significantly related to post salvage LT survival (multivariate analysis)

\begin{tabular}{lcccc}
\hline & \multicolumn{2}{c}{ Recurrence-free Survival } & \multicolumn{2}{c}{ Overall Survival } \\
\hline Variables & HR $(\mathbf{9 5 \%}$ CI) & $\boldsymbol{p}$ Value & HR (95\% CI) & $\boldsymbol{p ~ V a l u e ~}$ \\
\hline MVI presence at LR & $3.10[1.44-6.68]$ & 0.004 & $4.47[1.97-10.14]$ & $<0.001$ \\
Post-LR HCC recurrence $\leq 12$ months & $3.25[1.41-7.49]$ & 0.006 & $3.76[1.78-7.94]$ & 0.001 \\
Outside UCSF criteria at LT & $2.68[1.18-6.06]$ & 0.018 & & $4.71[2.08-10.71]$ \\
MELD score $\geq 15$ at LT & & & $<0.001$ \\
\hline
\end{tabular}

LT: liver transplantation; LR: liver resction; MVI: microinvascular invasion; HCC: hepatocellular carcinoma; UCSF:

University of California San Francisco; MELD: model for end-stage liver disease; HR: hazard ratio; CI: confidence interval. 
also reported that HCC recurrence within the 8 months following initial LR and a high serum AFP level ( $>$ $200 \mathrm{ng} / \mathrm{mL}$ ) at salvage LT were independent risk factors for post-salvage LT HCC recurrence [24]. A pre-LT AFP level > $200 \mathrm{ng} / \mathrm{mL}$ was also analyzed in our study and determined to be an unfavorable risk factor.

Several previous studies have recommended the use of salvage LT prior to HCC recurrence in patients with unfavorable risk factors [9, 19, 27]. For example, Fuks et al. recommended performing salvage LT before

(a)

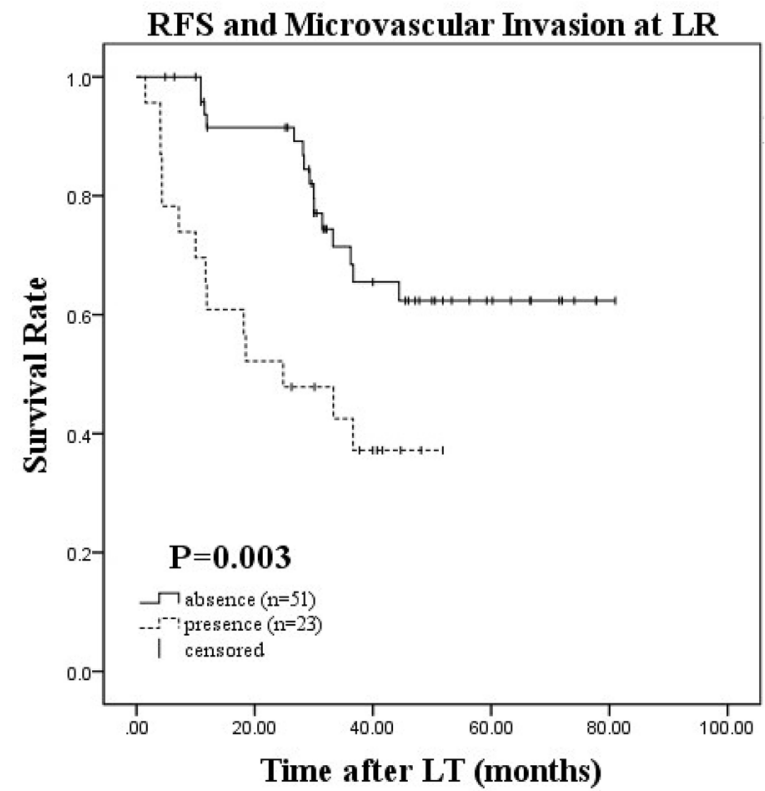

HCC recurrence in patients with $\geq 3$ of the five pejorative factors (MVI, satellite nodules, tumor $>3 \mathrm{~cm}$, poorly differentiated tumor, and liver cirrhosis) [9]. However, the main consideration of Fuks et al. was the high risk of nontransplantability after initial LR rather than long-term post-LT patient outcomes. Whether patients with all of these risk factors should undergo salvage LT prior to HCC recurrence is controversial.

Another recent study conducted by FerrerFabrega et al. proposed a waiting time of $>6$ months between resection and enlistment for transplant, even

(b)

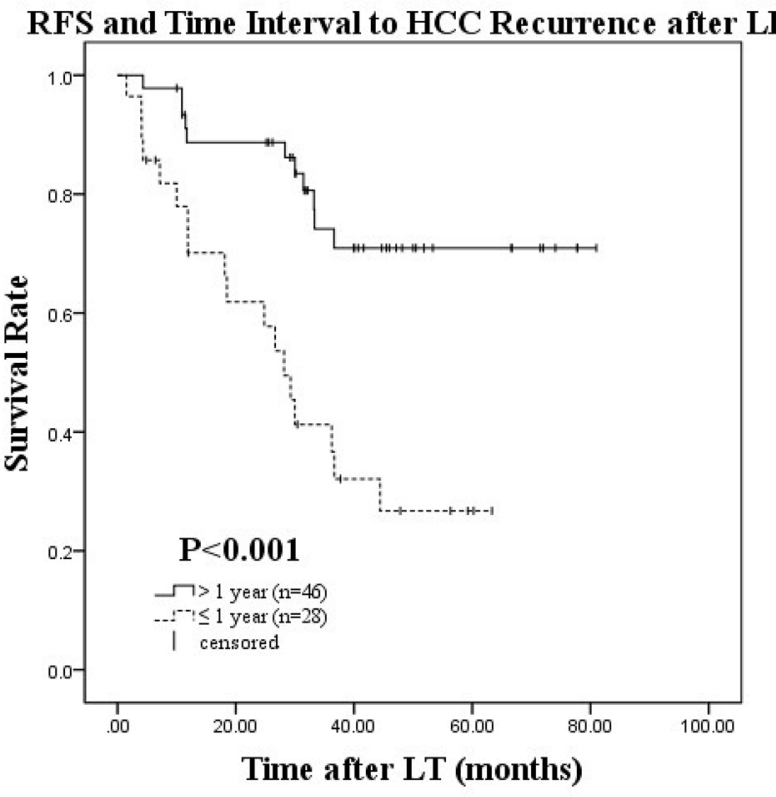

(c)

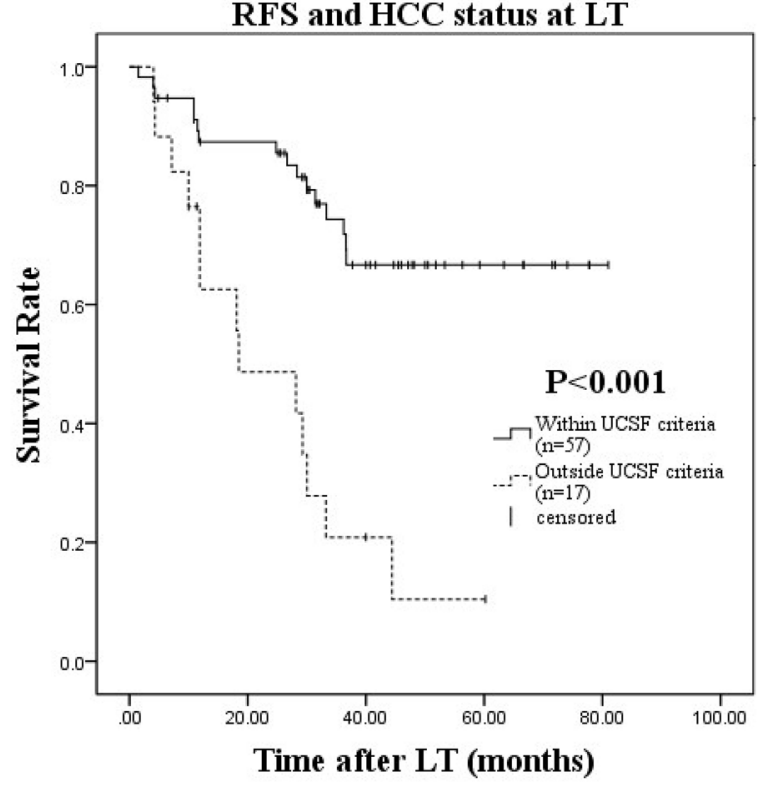

Figure 1: Recurrence-free survival after salvage LT and microvascular invasion at LR a. time interval to HCC recurrence after $\mathrm{LR}$ b. HCC status at LT c. 
in patients at high risk or recurrence (MVI and/or additional nodules or satellites), such that they could identify patients with less aggressive tumors and avoid performing LTs on patients with aggressive tumors (associated with a high risk of post-LT HCC recurrence and decreased survival) [27]. They recommend the use of salvage LT before recurrence in high-risk patients but not in patients with biologically aggressive tumors, in order to avoid disease recurrence with a dismal prognosis post-LT. Nonetheless, our study focused

(a)

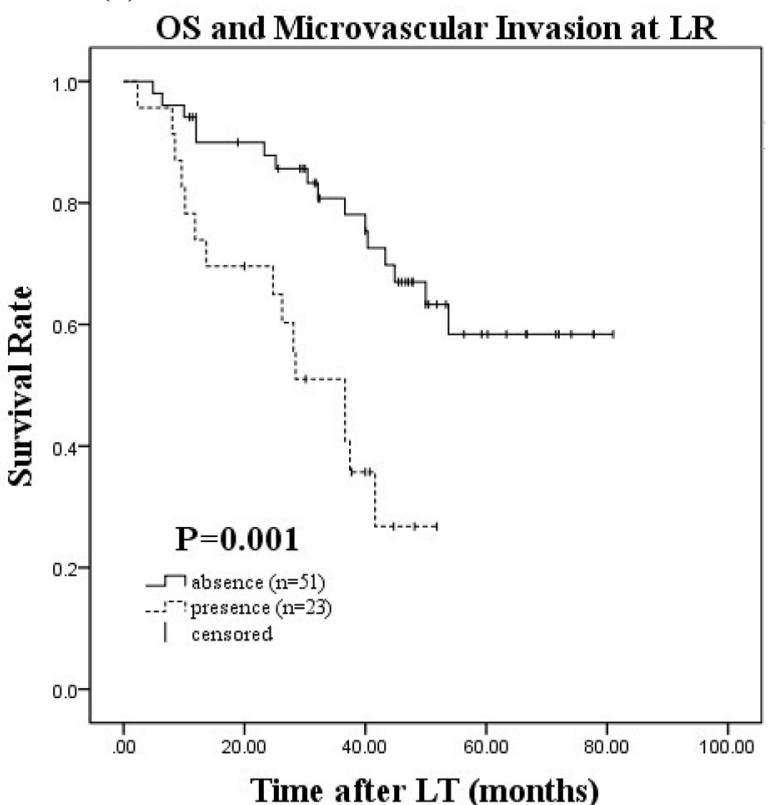

(c) on the time point of recurrence detected after LR to identify the most appropriate candidates for salvage LT considering the limited donor pool. In this regard, our results were consistent with those of Ferrer-Fabrega et al.

This study had several limitations. First, it was retrospective in nature and performed at a single center. Therefore, there were limitations as a result of the analysis of observational data. Our results require further validation by multi-center, prospective studies (b)

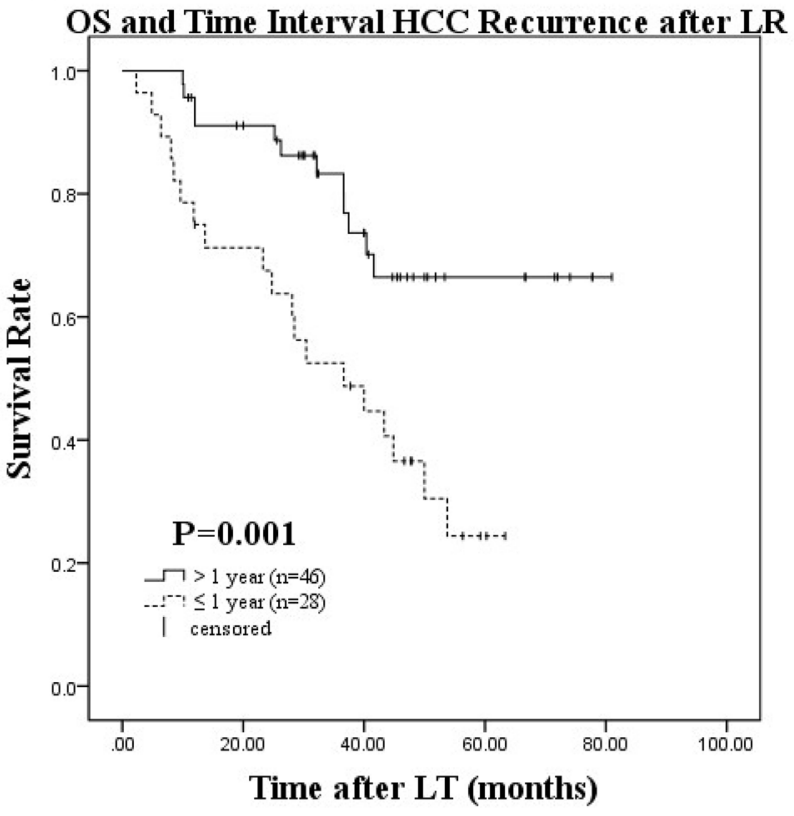

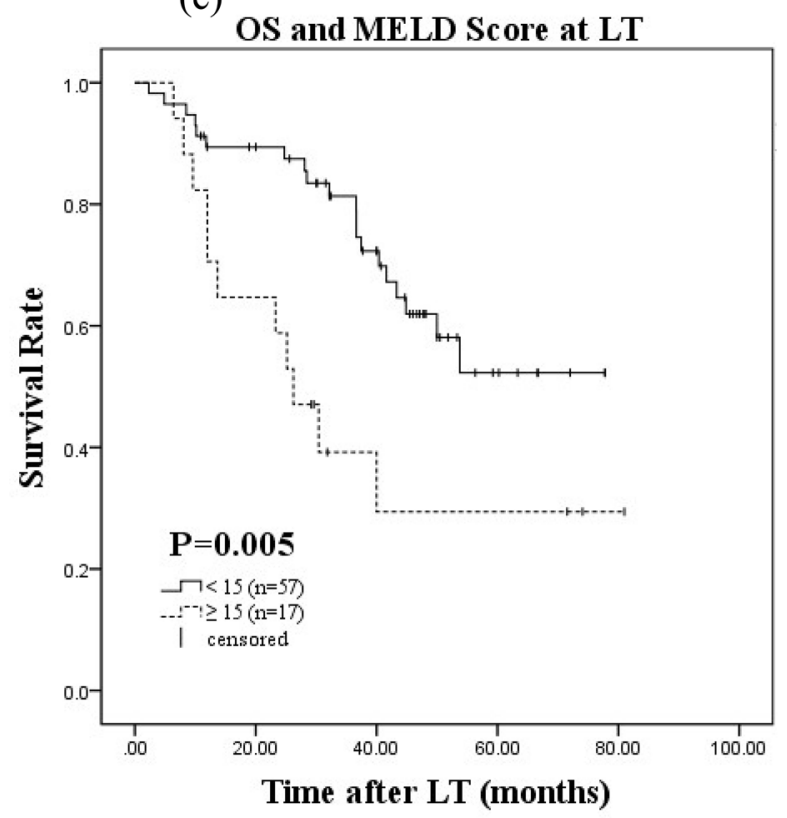

Figure 2: Overall survival after salvage $\mathrm{LT}$ and microvascular invasion at $\mathrm{LR}$ a. time interval to $\mathrm{HCC}$ recurrence after LR b. MELD score at LT c. 
Table 6: Prognosis of patients after salvage LT according to the number of risk factors

\begin{tabular}{|c|c|c|c|c|c|c|c|c|}
\hline \multirow[t]{2}{*}{$\begin{array}{l}\text { Risk Factor } \\
\text { Number }\end{array}$} & \multirow[t]{2}{*}{$\begin{array}{l}\text { Patients } \\
\text { (n) }\end{array}$} & \multirow[t]{2}{*}{$\begin{array}{c}\text { Recurrence } \\
\text { n (\%) }\end{array}$} & \multicolumn{3}{|c|}{$\begin{array}{l}\text { Recurrence-free } \\
\text { Survival (\%) } \\
\end{array}$} & \multirow[t]{2}{*}{$\begin{array}{l}\text { Mean Recurrence-free Survival } \\
\text { (95\%CI, months) }\end{array}$} & \multicolumn{2}{|c|}{$\begin{array}{l}p \text { Value (log- } \\
\text { rank test) }\end{array}$} \\
\hline & & & $1 \mathrm{y}$ & $3 y$ & $5 y$ & & & \\
\hline 0 & 26 & $3(11.54 \%)$ & 92.1 & 87.5 & 87.5 & $73.24[64.96-81.52]$ & Ref. & Ref. \\
\hline 1 & 27 & $9(33.33 \%)$ & 87.7 & 59.1 & 52.5 & $45.74[36.97-54.52]$ & 0.026 & \\
\hline 2 & 14 & $10(71.43 \%)$ & 78.6 & 42.9 & 23.8 & $33.87[23.67-44.06]$ & $<0.001$ & $<0.001$ \\
\hline $3-4$ & 7 & $7(100 \%)$ & 28.6 & 0.00 & 0.00 & $10.59[6.15-15.03]$ & $<0.001$ & \\
\hline
\end{tabular}

LT: liver transplantation; Ref: reference; CI: confidence interval.

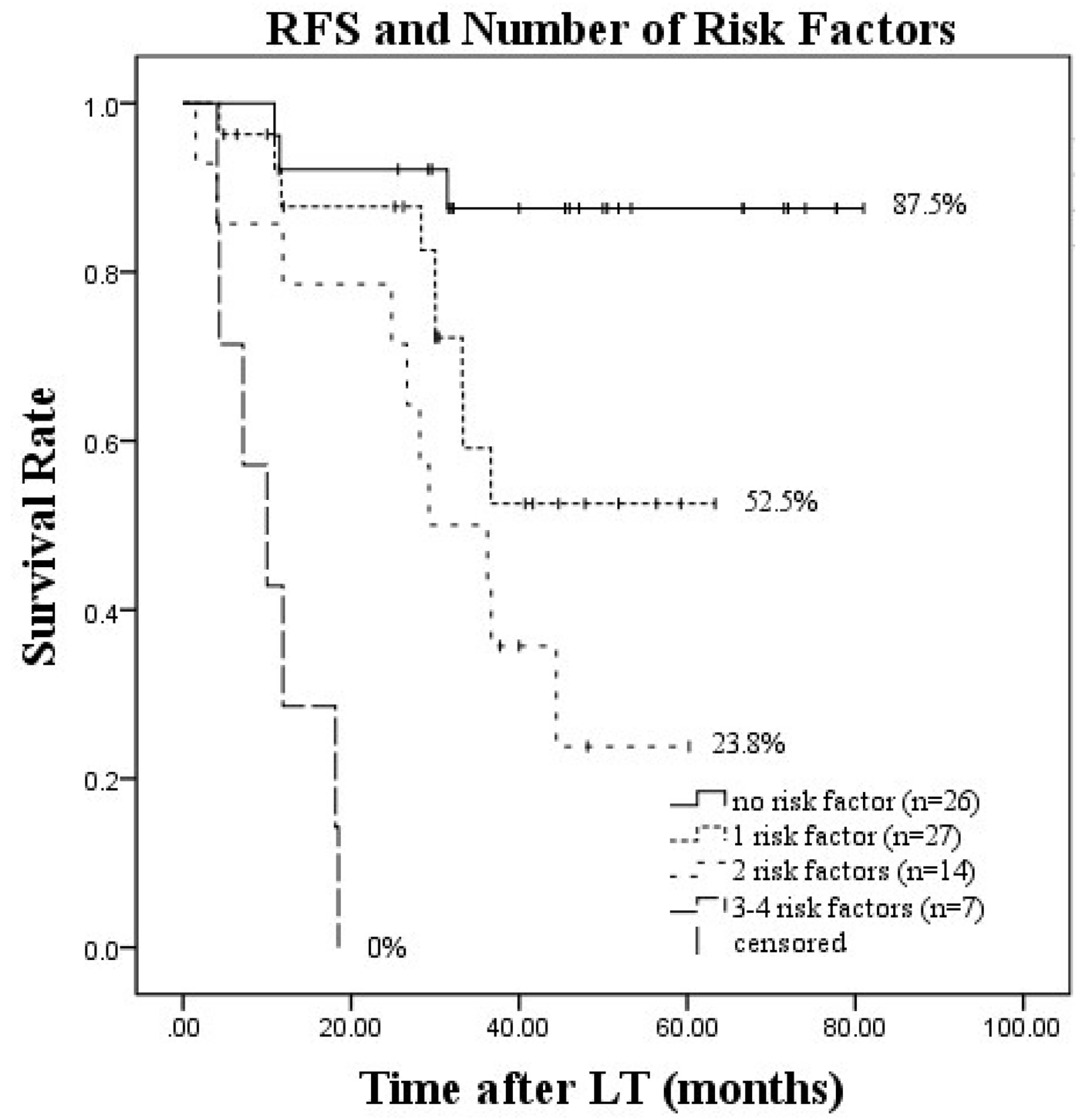

Figure 3: Recurrence-free survival after salvage $L T$ according to number of risk factors 
with larger sample sizes. Second, advances in surgical techniques, improved postoperative management, and more reasonable immunosuppressive therapy would have improved clinical prognosis after the study patient selection period, resulting in heterogeneity among the participants. Finally, the extended period over which patients were selected for inclusion was a limitation of the study.

In conclusion, we aimed to identify appropriate candidates for salvage LT at the time of HCC recurrence detected post-LR. In addition to the UCSF criteria at LT, we demonstrated that the presence of MVI at initial LR, a time interval to post-LR HCC recurrence of $\leq 12$ months, and a pre-LT AFP level $>200 \mathrm{ng} / \mathrm{mL}$ were independent risk factors for post-salvage LT HCC recurrence. Patients with no more than one risk factor had a 5-year RFS rate of $71.2 \%$, while those with $>2$ risk factors had a rate of $15.9 \%$. Our findings suggest that the priority of salvage LT should be given to patients with no more than one risk factor to avoid post-LT HCC recurrence and a dismal prognosis. Such a policy might improve salvage LT outcomes and facilitate the effective use of a limited donor organ supply.

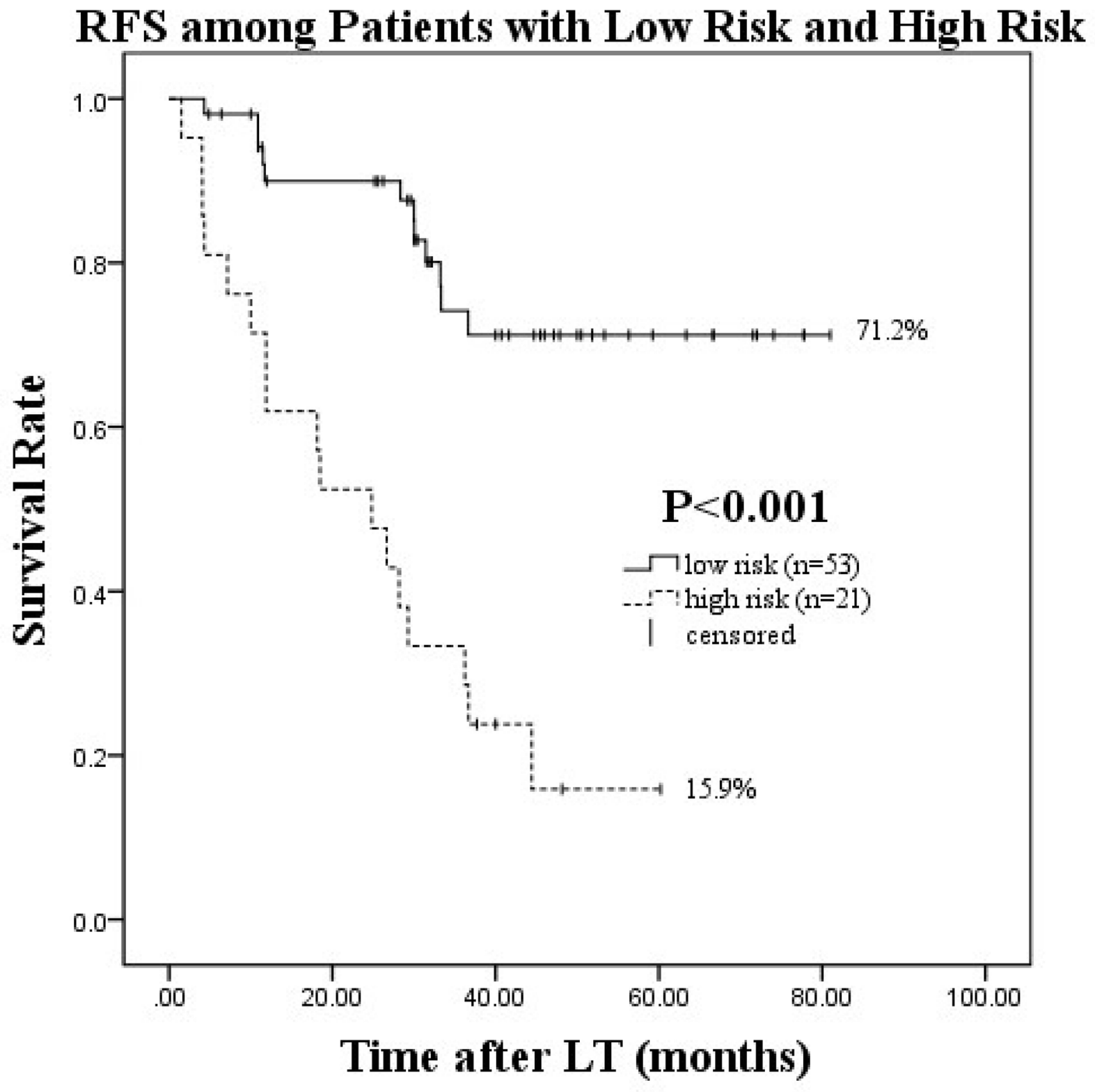

Figure 4: Recurrence-free survival after salvage LT in the low risk (0-1 risk factors) and high risk (2-4 risk factors) groups 


\section{MATERIALS AND METHODS}

\section{Ethical considerations}

This study was approved by the Institutional Review Board of the Shanghai General Hospital, Shanghai Jiao Tong University School of Medicine, and was conducted according to the 1964 Helsinki Declaration and its later amendments [28].

\section{Patients}

Between October 2001 and February 2013, a total of $89 \mathrm{HCC}$ patients with a previous history of LR underwent LT for the treatment of HCC recurrence at Shanghai General Hospital, Shanghai Jiao Tong University School of Medicine. All data were obtained from our prospectively maintained LT data base and patient medical records. The HCC diagnosis was confirmed by histopathology of explanted and resected tissue specimens.

Only patients with $\mathrm{HCC}$ who underwent salvage LT owing to intrahepatic recurrence after a previous LR were included in the study. The exclusion criteria were: (1) recurrence of other malignancies in addition to HCC such as cholangiocarcinoma $(\mathrm{n}=1)$; (2) tumor thrombosis of the main blood vessel trunk (portal vein and hepatic vein) $(\mathrm{n}=2)$; (3) having undergone LR or LT more than once $(n=3)$; and (4) a lack of precise pathology data $(n=9)$.

\section{Data collection}

Patient baseline and clinical data consisting of sex, blood type, underlying liver disease, preoperative AFP level at LR, HCC status according to the Milan criteria at LR, Edmondson grade at LR, presence of MVI at LR, time interval to post-LR HCC recurrence, age at LT, preoperative MELD score at LT [29], Child-Pugh grading at LT [30], preoperative AFP level at LT, HCC status according to the UCSF criteria at LT, TNM staging at LT (according to the International Union Against Cancer/American Joint Committee on Cancer criteria) [31], presence of MVI at LT, HCC recurrence, number of deaths, OS, and RFS were recorded. Surviving patients or those who died of non-HCC causes were censored at the date of their last visit or date of death.

\section{Tumor surveillance and treatment after LR and LT}

After LR and LT, follow-up included ultrasonography, computed tomography, or magnetic resonance imaging scans as well as measurement of serum AFP levels (every 3-4 months for the first 3 years, every 4-6 months for the next 3-5 years, and then every 6-12 months after 5 years) according to the protocol of the Ministry of Health of the People's Republic of China. Recurrence was defined as the appearance of new lesions with HCC features on imaging. Upon detection of recurrence after LR, positron emission tomography/ computed tomography, chest computed tomography, or bone scintigraphy was performed to exclude the existence of distant metastases. Post-LT recurrence was treated with locoregional therapies such as radiofrequency ablation, transarterial chemoembolization, radiotherapy, or a combination of these strategies whenever possible. Underlying liver diseases including chronic HBV, HCV, and autoimmune hepatitis were all treated appropriately pre- and post-LT. HBV recurrence was monitored by checking for the presence of HBV surface antigen and HBV DNA in serum at every follow-up visit.

The immunosuppressive regimens used post-LT included either basiliximab or steroids in addition to tacrolimus and mycophenolatemofetil (MMF). Tacrolimus treatment was contraindicated in patients with insufficient renal function (creatinine $>120 \mu \mathrm{mol} / \mathrm{L}$ or creatinine clearance $<40 \mathrm{~mL} / \mathrm{min})$. MMF was used in patients with relatively low serum tacrolimus levels as well as in those without pancytopenia (hematocrit $>26 \%$ and platelet count $>50,000$ cells $/ \mathrm{mm}^{3}$ ) [32]. When MMF was administered, tacrolimus was maintained at a lower blood concentration. Liver function as well as the blood concentrations of the immunosuppressants were monitored for dose adjustment purposes.

\section{Statistical analysis}

All of the statistical analyses were performed using the SPSS statistical software, Version 19.0 (SPSS Inc., Chicago, IL, USA). Continuous data were expressed as the mean \pm standard deviation (SD) or the median (range), while discrete variables were shown as frequencies. Categorical variables were compared using Pearson's $\chi^{2}$ test or Fisher's exact test, whereas continuous variables were calculated using Student's t-test or the Mann-Whitney test. All variables were dichotomized for analysis. The logistic regression model was applied to investigate post-LT predictors of HCC recurrence. Survival time started at the time of the LT procedure. Survival rates were assessed using Kaplan-Meier analysis and differences between subgroups were compared using the log-rank test. The clinicopathological characteristics of the patients were calculated using the Cox proportional hazard regression model for RFS and OS. The final models were determined by placing all variables with $P$ values $<$ 0.10 from the univariate analysis into the multivariate Cox regression analysis. Statistical significance was established as $P<0.05$.

\section{CONFLICTS OF INTEREST}

The authors declare no conflicts of interest. 


\section{REFERENCES}

1. Forner A, Llovet JM, Bruix J. Hepatocellular carcinoma. Lancet. 2012; 379:1245-1255.

2. Torre LA, Bray F, Siegel RL, Ferlay J, Lortet-Tieulent J, Jemal A. Global cancer statistics, 2012. CA. 2015; 65:87-108.

3. Mazzaferro V, Regalia E, Doci R, Andreola S, Pulvirenti A, Bozzetti F, Montalto F, Ammatuna M, Morabito A, Gennari L. Liver transplantation for the treatment of small hepatocellular carcinomas in patients with cirrhosis. The New England journal of medicine. 1996; 334:693-699.

4. Poon RT, Fan ST, Lo CM, Liu CL, Wong J. Difference in tumor invasiveness in cirrhotic patients with hepatocellular carcinoma fulfilling the Milan criteria treated by resection and transplantation: impact on long-term survival. Annals of surgery. 2007; 245:51-58.

5. Concejero A, Chen CL, Wang CC, Wang SH, Lin CC, Liu YW, Yang CH, Yong CC, Lin TS, Jawan B, Huang TL, Cheng YF, Eng HL. Living donor liver transplantation for hepatocellular carcinoma: a single-center experience in Taiwan. Transplantation. 2008; 85:398-406.

6. Ota K, Teraoka S, Kawai T. Donor difficulties in Japan and Asian countries. Transplantation proceedings. 1995; 27:83-86.

7. Majno PE, Sarasin FP, Mentha G, Hadengue A. Primary liver resection and salvage transplantation or primary liver transplantation in patients with single, small hepatocellular carcinoma and preserved liver function: an outcomeoriented decision analysis. Hepatology. 2000; 31:899-906.

8. Lim KC, Chow PK, Allen JC, Siddiqui FJ, Chan ES, Tan SB. Systematic review of outcomes of liver resection for early hepatocellular carcinoma within the Milan criteria. The British journal of surgery. 2012; 99:1622-1629.

9. Fuks D, Dokmak S, Paradis V, Diouf M, Durand F, Belghiti J. Benefit of initial resection of hepatocellular carcinoma followed by transplantation in case of recurrence: an intention-to-treat analysis. Hepatology. 2012; 55:132-140.

10. Del Gaudio M, Ercolani G, Ravaioli M, Cescon M, Lauro A, Vivarelli M, Zanello M, Cucchetti A, Vetrone G, Tuci F, Ramacciato G, Grazi GL, Pinna AD. Liver transplantation for recurrent hepatocellular carcinoma on cirrhosis after liver resection: University of Bologna experience. American journal of transplantation. 2008; 8:1177-1185.

11. Guerrini GP, Gerunda GE, Montalti R, Ballarin R, Cautero N, De Ruvo N, Spaggiari M, Di Benedetto F. Results of salvage liver transplantation. Liver international. 2014; 34:e96-e104.

12. Chan DL, Alzahrani NA, Morris DL, Chua TC. Systematic review of efficacy and outcomes of salvage liver transplantation after primary hepatic resection for hepatocellular carcinoma. Journal of gastroenterology and hepatology. 2014; 29:31-41.
13. Hu Z, Zhou J, Xu X, Li Z, Zhou L, Wu J, Zhang M, Zheng $\mathrm{S}$. Salvage liver transplantation is a reasonable option for selected patients who have recurrent hepatocellular carcinoma after liver resection. PloS one. 2012; 7:e36587.

14. Liu F, Wei Y, Wang W, Chen K, Yan L, Wen T, Zhao $\mathrm{J}, \mathrm{Xu}$ M, Li B. Salvage liver transplantation for recurrent hepatocellular carcinoma within UCSF criteria after liver resection. PloS one. 2012; 7:e48932.

15. Wu L, Hu A, Tam N, Zhang J, Lin M, Guo Z, He X. Salvage liver transplantation for patients with recurrent hepatocellular carcinoma after curative resection. PloS one. 2012; 7:e41820.

16. Toso C, Mentha G, Majno P. Liver transplantation for hepatocellular carcinoma: five steps to prevent recurrence. American journal of transplantation. 2011; 11:2031-2035.

17. Yao FY, Ferrell L, Bass NM, Watson JJ, Bacchetti P, Venook A, Ascher NL, Roberts JP. Liver transplantation for hepatocellular carcinoma: expansion of the tumor size limits does not adversely impact survival. Hepatology. 2001; 33:1394-1403.

18. Mazzaferro V, Llovet JM, Miceli R, Bhoori S, Schiavo M, Mariani L, Camerini T, Roayaie S, Schwartz ME, Grazi GL, Adam R, Neuhaus P, Salizzoni M, et al. Predicting survival after liver transplantation in patients with hepatocellular carcinoma beyond the Milan criteria: a retrospective, exploratory analysis. The Lancet Oncology. 2009; 10:35-43.

19. Sala M, Fuster J, Llovet JM, Navasa M, Sole M, Varela M, Pons F, Rimola A, Garcia-Valdecasas JC, Bru C, Bruix J, Barcelona Clinic Liver Cancer G. High pathological risk of recurrence after surgical resection for hepatocellular carcinoma: an indication for salvage liver transplantation. Liver transplantation. 2004; 10:1294-1300.

20. Lee HS, Choi GH, Joo DJ, Kim MS, Choi JS, Kim SI. The clinical behavior of transplantable recurrent hepatocellular carcinoma after curative resection: implications for salvage liver transplantation. Annals of surgical oncology. 2014; 21:2717-2724.

21. Choi GH, Kim DH, Kang CM, Kim KS, Choi JS, Lee WJ, Kim BR. Prognostic factors and optimal treatment strategy for intrahepatic nodular recurrence after curative resection of hepatocellular carcinoma. Annals of surgical oncology. 2008; 15:618-629.

22. Ding T, Xu J, Zhang Y, Guo RP, Wu WC, Zhang SD, Qian CN, Zheng L. Endothelium-coated tumor clusters are associated with poor prognosis and micrometastasis of hepatocellular carcinoma after resection. Cancer. 2011; 117:4878-4889.

23. Hou YF, Wei YG, Yang JY, Wen TF, Xu MQ, Yan LN, Li B, Chen KF. Microvascular invasion patterns affect survival in hepatocellular carcinoma patients after second hepatectomy. The Journal of surgical research. 2016; 200:82-90.

24. Lee S, Hyuck David Kwon C, Man Kim J, Joh JW, Woon Paik S, Kim BW, Wang HJ, Lee KW, Suh KS, Lee SK. Time of hepatocellular carcinoma recurrence 
after liver resection and alpha-fetoprotein are important prognostic factors for salvage liver transplantation. Liver transplantation. 2014; 20:1057-1063.

25. Poon RT, Fan ST, Ng IO, Lo CM, Liu CL, Wong J. Different risk factors and prognosis for early and late intrahepatic recurrence after resection of hepatocellular carcinoma. Cancer. 2000; 89:500-507.

26. Hu Z, Zhou J, Li Z, Xiang J, Qian Z, Wu J, Zhang M, Zheng S. Time interval to recurrence as a predictor of overall survival in salvage liver transplantation for patients with hepatocellular carcinoma associated with hepatitis B virus. Surgery. 2015; 157:239-248.

27. Ferrer-Fabrega J, Forner A, Liccioni A, Miquel R, Molina V, Navasa M, Fondevila C, Garcia-Valdecasas JC, Bruix J, Fuster J. Prospective validation of "ab initio" liver transplantation in hepatocellular carcinoma upon detection of risk factors for recurrence after resection. Hepatology. 2015.

28. World Medical Association declaration of Helsinki. Recommendations guiding physicians in biomedical research involving human subjects. Jama. 1997; 277:925-926.
29. Malinchoc M, Kamath PS, Gordon FD, Peine CJ, Rank J, ter Borg PC. A model to predict poor survival in patients undergoing transjugular intrahepatic portosystemic shunts. Hepatology. 2000; 31:864-871.

30. Pugh RN, Murray-Lyon IM, Dawson JL, Pietroni MC, Williams R. Transection of the oesophagus for bleeding oesophageal varices. The British journal of surgery. 1973; 60:646-649.

31. Edge SB, Compton CC. The American Joint Committee on Cancer: the 7th edition of the AJCC cancer staging manual and the future of TNM. Annals of surgical oncology. 2010; 17:1471-1474.

32. Xing T, Huang L, Yu Z, Zhong L, Wang S, Peng Z. Comparison of steroid-free immunosuppression and standard immunosuppression for liver transplant patients with hepatocellular carcinoma. PloS one. 2013; 8:e71251. 